\section{DO ADIPOSITY INDICES INFLUENCE VO2 MAX WITHIN NORIMAL LIMITS? FITNESS VS. FATNESS}

KEY WORDS: Waist circumference, Conicity index, $\mathrm{ABSI}, \mathrm{VO}_{2}$ max, visceral adiposity, aerobic capacity

\section{Haroon Rashid} M. Hattiwale

\section{Shaheenkousar}

H. Hattiwale*

Salim $\mathbf{A}$.

Dhundasi

Mohammad

Muzammil

Ahmed

Mohammed

Nazeer

Khwaja Mohd

\section{Amir}

Associate Professor, Department of Physiology, Basic Medical Sciences, College of Medicine 11952, Majmaah University, Al Majmaah, Kingdom of Saudi Arabia.

Associate Professor, Department of Physiology, Basic Medical Sciences, College of Medicine 11952, Majmaah University, Al Majmaah, Kingdom of Saudi Arabia. *Corresponding Author

Professor, Department of Physiology, Al Ameen Medical College, Bijapur, Karnataka,586108, India.

Assistant Professor, Department of Anatomy, Basic Medical Sciences, College of Medicine 11952, Majmaah University, Al Majmaah, Kingdom of Saudi Arabia.

Assistant Professor, Department of Anatomy, Basic Medical Sciences, College of Medicine 11952, Majmaah University, Al Majmaah, Kingdom of Saudi Arabia.

Assistant Professor, Department of Physiology, Basic Medical Sciences, College of Medicine 11952, Majmaah University, Al Majmaah, Kingdom of Saudi Arabia.

Introduction: The global burden of obesity doubled in children, tripled in adolescents and adults between 1975 and 2016. The central obesity is strongly associated with risk of type 2 diabetes and coronary artery diseases. Therefore, it is important to screen/detect obesity and its complications in early stages. Aim: of this study is to evaluate early markers of adult overweight/obesity, visceral obesity and aerobic fitness in young, healthy men, and also to find out association between visceral obesity and $\mathrm{VO}_{2}$ max as adequate research data is not available in this field. Methods: Forty young, healthy adult men (aged 18-40 years) were randomly selected from the population of Vijayapura, Karnataka, India, in this study. The anthropometric parameters; weight, height, BMI, BSA, waist circumference and hip circumference were measured. The waist-hip ratio, Conicity index and A Body surface Index were calculated. $\mathrm{VO}_{2}$ max was measured by Rockport 1-Mile walk test. A series of Pearson's correlation tests were performed to find out the influence of visceral adiposity indices on aerobic fitness. Results: All anthropometric parameters and adiposity indices measured in our study were within the normal range. A significant positive correlation was observed between age vs conicity index and age vs ABSI. We also found a significant negative correlation between adiposity indices and $\mathrm{VO}_{2}$ max in young healthy adult males. Conclusion: Waist circumference, waist-hip ratio, conicity index and ABSI can be considered as useful anthropometric tools to diagnose central/visceral obesity in healthy men with normal BMI. Total fat and visceral fat may probably have some negative influence on cardiorespiratory function and aerobic fitness.

\section{INTRODUCTION}

Obesity is defined as an excess or abnormal accumulation of body fat. The worldwide prevalence of childhood obesity nearly doubled, tripled in adolescents and adults between 1975 and 2016. It is one of the most neglected global public health issues ${ }^{[1]}$. Currently, BMI is the most widely used anthropometric tool for diagnosing and rating obesity in human subjects. However, BMI does not differentiate between muscle and fat mass, unable to detect visceral adiposity at individual level. Therefore, WHO has suggested that it is more appropriate to evaluate abdominal obesity for assessing cardiometabolic diseases and mortality risk ${ }^{[2]}$. The magnetic resonance imaging and computed tomography are accurate methods to measure visceral fat. However, they can not be used routinely in clinical follow up or epidemiological studies. The anthropometric parameters and adiposity indices are simple, accurate and economical surrogate markers of visceral adiposity ${ }^{[3]}$. A study published in Nature; the authors have concluded that waist circumference is vital tool to measure visceral adiposity in a clinical setup ${ }^{[4]}$. A body Surface Index (ABSI), Conicity index and waist-hip ratio are other simple adiposity indices used to measure visceral fat ${ }^{[3]}$. Low level of aerobic fitness and physical inactivity is another independent risk factor strongly correlated with morbidity and mortality; and in contrary good cardiorespiratory fitness reduces both long term morbidity and mortality ${ }^{[5]}$. Therefore, we have designed this study to evaluate early markers of adult overweight/obesity, visceral obesity and aerobic fitness in young, healthy men of Vijayapura, Karnataka, India; and also to find out association between visceral obesity parameters with $\mathrm{VO}_{2}$ max as adequate research data is not available in this field.

\section{Materials And Methods} Inclusion criteria:

40 young, healthy, adult, males aged 18-40 years were randomly selected from the population of Vijayapura, Karnataka, India, for this study. The entire protocol was explained in their local language and written consent was obtained from the volunteers. Exclusion criteria: Men with musculoskeletal diseases, cardio respiratory, endocrine disorders, anemia and diabetes mellitus are excluded from this study. The selected subjects were requested to visit the laboratory in the morning hours with a light breakfast. The height was measured in centimeter on a calibrated stadiometer. The weight of the participants was recorded in kilogram on weighing scale, the BMI was calculated by Quetelet's index from body weight $(\mathrm{Kg}) /(\text { height } \mathrm{m})^{2}$. The waist circumference (WC) was measured in centimeters in a standing position with a flexible measuring tape at midpoint between lower rib margin and upper part of iliac crest; hip circumference was measured in centimeters at the level of trochanters the largest horizontal circumference around the 
buttocks and waist -hip ratio (WHR) was calculated ${ }^{[6]}$. A Body Surface Area was calculated by using this formula, ABSI= $\mathrm{WC}(\mathrm{m}) /\left[\mathrm{BMI}^{2 / 3} \times \operatorname{Height}(\mathrm{m})^{1 / 2}\right] ;{ }^{[7]}$. A conicity index was calculated by: CI = WC (m)/ $[0.109 \mathrm{x} V$ \{Bodyweight (kg)/ Height $(\mathrm{m})\}]$ where 0.109 is a constant ${ }^{[8]}$.In both the equations, weight was expressed in kilograms and height and waist circumference (WC) in meters. $\mathrm{VO}_{2}$ max was measured by Rockport l-Mile walk test ${ }^{[9]}$. The subjects were asked to walk individually as fast as possible (not to run) for a distance of one mile $(1.6 \mathrm{~km})$ on a flat surface. Pulse rate was recorded immediately after walk. The $\mathrm{VO}_{2}$ max was determined by substituting pulse rate in the following formula for males; $\mathrm{VO}_{2}$ $\max (\mathrm{ml} / \mathrm{kg} / \mathrm{min})=.139.168-(0.388 \times \mathrm{age})-(0.077 \times$ weight in lb) $-(3.265 \times$ walk time in minutes $)-(0.156 \times$ heart rate $)+$ 6.318. The experimental protocol was approved by Institutional Ethical Committee as per the guidelines of declaration of Helsinki 1975.

All the values of age, anthropometric parameters and adiposity indices were expressed as mean \pm SD. Pearson's correlation analysis was done to determine relationship between adiposity indices and $\mathrm{VO}_{2}$ max by using SPSS software. The level of significance was set at $p<0.05$.

\section{RESULTS}

Table 1 depicts mean \pm SD of age, physical anthropometric parameters and adiposity indices of all the subjects in this study. The mean values of all the parameters were found to be within normal range.

Table 1: Age, physical anthropometric parameters, body adiposity indices and $\mathrm{VO}_{2}$ max of all the subjects in our study.

\begin{tabular}{|l|l|}
\hline Parameters & Mean $\mathbf{\text { SD }}(\mathbf{n}=40)$ \\
\hline Age $($ years $)$ & $24.175+8.277$ \\
\hline Height $(\mathrm{cm})$ & $169.575+6.880$ \\
\hline Weight $(\mathrm{kg})$ & $64.42+14.17$ \\
\hline BSA $\left(\mathrm{m}^{2)}\right.$ & $1.73 \pm 0.222$ \\
\hline BMI $\left(\mathrm{kg} / \mathrm{m}^{2}\right)$ & $22.29 \pm 3.903$ \\
\hline Waist circumference $(\mathrm{cm})$ & $76.5 \pm 11.071$ \\
\hline Hip circumference $(\mathrm{cm})$ & $89.2 \pm 8.29$ \\
\hline Waist Hip Ratio & $0.85 \pm 0.06$ \\
\hline Conicity index & $1.141 \pm 0.073$ \\
\hline A Body Surface Index & $0.0742 \pm 0.004$ \\
\hline VO $_{2}$ max ml/Kg/min & $61.34 \pm 5.39$ \\
\hline
\end{tabular}

Table 2 shows Pearson's correlation coefficient values between different parameters. Our study shows nonsignificant positive correlation between age and BMI, however we found statical significant positive correlation between age vs conicity index and age vs ABSI. Our study further shows significant negative correlation between adiposity indices; BMI, waist circumference, hip circumference, waist hip ratio, ABSI and conicity index with $\mathrm{VO}_{2} \max$.

Table 2: The Correlation values of adiposity indices vs. VO2 $\mathrm{max} \mathrm{ml} / \mathrm{kg} / \mathrm{min}$

\begin{tabular}{|l|l|l|}
\hline Parameters & \multicolumn{2}{l|}{$\begin{array}{l}\text { Pearson's correlation } \\
\text { coefficient }\end{array}$} \\
\hline & r-value & p- value \\
\hline Age vs BMI & +0.171 & $\mathrm{P}<0.292$ \\
\hline Age vs Conicity index & +0.361 & $\mathrm{P}<0.022^{*}$ \\
\hline Age vs. ABSI & +0.327 & $\mathrm{P}<0.039^{*}$ \\
\hline BMI vs $\mathrm{VO}_{2}$ max & -0.6568 & $\mathrm{P}<0.001^{*}$ \\
\hline Waist circumference vs $\mathrm{VO}_{2} \max$ & -0.7096 & $\mathrm{P}<0.001^{*}$ \\
\hline Hip circumference vs. $\mathrm{VO}_{2} \max$ & -0.6428 & $\mathrm{P}<0.001^{*}$ \\
\hline Waist Hip Ratio vs $\mathrm{VO}_{2} \mathrm{max}$ & -0.5702 & $\mathrm{P}<0.001^{*}$ \\
\hline Conicity index vs $\mathrm{VO}_{2} \max$ & -0.597 & $\mathrm{P}<0.001^{*}$ \\
\hline ABSI vs $\mathrm{VO}_{2} \max$ & -0.3646 & $\mathrm{P}<0.021^{*}$ \\
\hline
\end{tabular}

$\mathrm{n}=40 \quad$ Significant $* \mathrm{p}<0.05$

|www.worldwidejournals.com $\mid$
Figure-1 clearly depicts negative but statical significant correlation between ABSI and $\mathrm{Vo}_{2} \max \AA=-0.3646, \mathrm{p}<0.021$ ) and figure-2 depicts statical significant negative correlation between conicity index andVO2max $(r=-0.597, p<0.001)$.

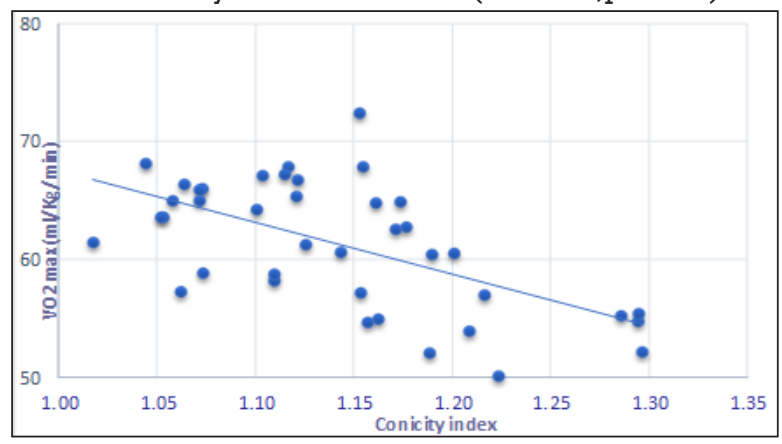

Figure 1: Relationship between Conicity index and VO2max: $(n=40)$; Pearson's correlation coefficient $(r)$ is$0.597, \mathrm{P}<0.001$

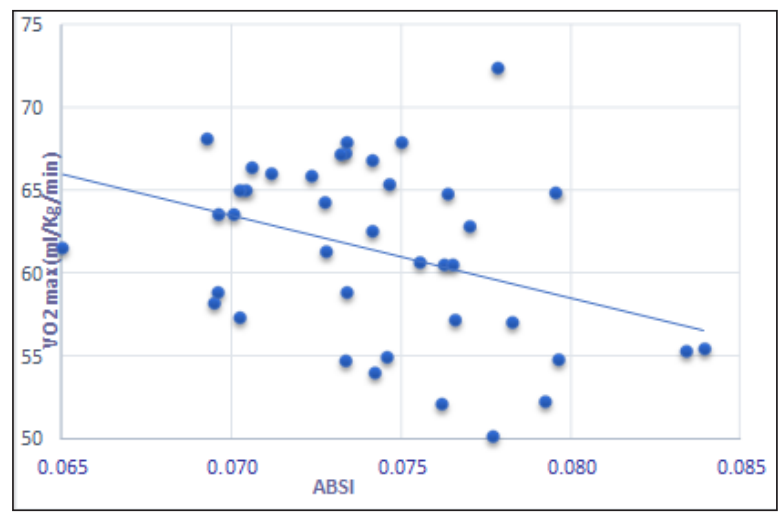

Figure.2: Relationship between $A B S I$ and VO2max $(n=40)$; Pearson's correlation coefficient $(r)$ is $0.3646, P<0.021$

\section{DISCUSSION}

The average values of anthropometric parameters measured in our study are within normal range. The normal range of BSA is $1.90-1.92^{[10]}$ and BMI is $18.5-24.9 \mathrm{Kg} / \mathrm{m}^{2}$. It signifies good current health status, life style and adequate nutrition. BMI is a useful tool for clinicians and researchers to quickly evaluate the risks associated with obesity. However BMI has some major limitations; It does not differentiate between fat and muscle mass, and unable to discriminate between peripheral and central obesity. Thus, BMI alone cannot be used as a research tool for assessing adiposity. It has been reported that within normal range of BMI there is distinct variability in adiposity with age and gender ${ }^{[1]}$. The older individual is likely to have more body fat than a younger one with an equal $\mathrm{BMI}$ as it does not consider age or gender in calculation. In a study conducted by Li B, et al on Chinese population found even in those individuals who are not overweight/ obese in adolescence, the increase in their BMI during growth and development to early adulthood was predictive of metabolic syndrome in their early adult life ${ }^{[12]}$. In our study age shows significant positive correlation with conicity index and ABSI which may probably be due to fat redistribution and increase in abdominal fat with age. This finding is supported by other researchers who have reported age-related changes in body composition like increase of total fat more specifically increase of visceral fat in abdomen and the decrease of lower body subcutaneous fat ${ }^{[13]}$. It is not just presence of excess fat but its distribution in the body that is corelated with cardiometabolic morbidity and mortality. An adverse fat distribution marked by increased abdominal and decreased leg fat increases the risk of atherosclerotic cardiovascular disease independent of total body fat mass. The visceral fat induces metabolic dysregulation that may induce inflammation, endothelial dysfunction and insulin resistance 
and it is considered as chief risk factor for hypertension, cardiovascular diseases and type 2 diabetes. The visceral adipose tissue can be evaluated indirectly by measuring central obesity ${ }^{[14]}$. The adiposity indices; waist circumference, hip circumference, waist-hip ratio, conicity index and ABSI measured in our study are also within normal limits. The normal range of waist circumference in men is $83-98 \mathrm{~cm}$, hip circumference $94-105 \mathrm{~cm}$, waist-hip ratio is $0.87-0.99^{[2]}$, conicity index $1.00-1.73^{[8]}$, and ABSI is $0.081-0.090^{[7]}$.

According to waist- hip ratio rating scale for men, the risk for cardiovascular and metabolic diseases are classified as high risk (> 1.0), moderately high risk (0.9 to 1.0) and optimal low risk $(<0.9)$ respectively ${ }^{[15]}$. The normal adiposity indices indicate normal fat mass and normal fat distribution in our study. $\mathrm{VO}_{2} \max$ is a measure of aerobic fitness and endurance. There are many factors that can influence $\mathrm{VO}_{2}$ max like age, sex, genetic and racial factors, physical training, and body composition. The $\mathrm{VO}_{2}$ max measured in our study is good. The interesting finding in our study is, despite being all values of anthropometric parameters, adiposity indices and $\mathrm{VO}_{2}$ max within normal range; we found a significant negative correlation between adiposity indices and $\mathrm{VO}_{2}$ max in young healthy adult males. Our findings are corroborated with Radovanovic $\mathrm{S}$ et al and Albornoz-Guerrero J et al ${ }^{[16-17]}$. It may probably be due to the fact that excess weight in our subjects has not only acted as passive load during submaximal exercise but may also be due to other physiological reasons like altered muscular and vascular homeostatic mechanisms ${ }^{[18]}$ or may be due to relatively less efficient cardiac performance during exercise ${ }^{[19]}$. The fatness was negatively correlated with aerobic fitness in our study. Obesity and physical inactivity both are modifiable risk factors and can be modified by effective physical exercise, health promotion by adopting active life style and a balance diet with restricted calories may help to achieve better cardiorespiratory fitness and lesser fatness.

\section{CONCLUSION}

Our study supports the following conclusions: Waist circumference, waist-hip ratio, conicity index and ABSI can be considered as useful anthropometric tools to recognize people with central/visceral obesity despite their normal BMI in healthy adult men, who are not apparently overweight or obese. A significant negative correlation between BMI, waist circumference, waist-hip ratio, conicity index, ABSI and $\mathrm{VO}_{2}$ max indicate that total fat and visceral fat may probably have some negative influence on cardiorespiratory function and aerobic fitness in young healthy adults. Consequently, further research is needed to explain the complex relationship between visceral adiposity and aerobic fitness in large sample size.

\section{REFERENCES}

1. Di Cesare,M., Sori ,M., Bovet, P. et al.The epidemiological burden of obesity in childhood: a worldwide epidemic requiring urgent action. BMC Med. 2019; 17(1):212.https://doi.org/10.1186/s12916-019-1449-8

2. Molarius A, Seidell JC, Sans S, Tuomilehto J, Kuulasmaa K. Waist and hip circumferences, and waist-hip ratio in 19 populations of the WHO MONICA Project. Int J Obes Relat Metab Disord. 1999;23(2):1 16-125. doi:10.1038/sj.ijo. 0800772

3. Roriz C, Karla A, Passos S, et al. Anthropometric clinical indicators in the assessment of visceral obesity: An update. Nutr. clin. diet. hosp. 2016; 36(2):168-179 DOI: 10.12873/362carneirororiz.

4. Ross R, Neeland IJ, Yamashita S, et al. Waist circumference as a vital sign in clinical practice: a Consensus Statement from the IAS and ICCR Working Group on Visceral Obesity. Nat Rev Endocrinol. 2020;16(3):177-189. Group on Visceral Obesity.

5. Mandsager K, Harb S, Cremer P, Phelan D, Nissen SE, Jaber W. Association of Cardiorespiratory Fitness With Long-term Mortality Among Adults Undergoing Exercise Treadmill Testing. JAMA Netw Open. 2018;1(6): e 183605. doi:10.1001/jamanetworkopen.2018.3605

6. Agbo HA, Zoakah AI, Isichei CO, Sagay AS, Achenbach CJ, Okeahialam BN. Cardiovascular Anthropometry: What Is Best Suited for Large-Scale Population Screening in Sub-Saharan Africa?. Front Cardiovasc Med. Population Screening in Sub-Saharan Africa?. Front Cardiovas

7. Gomez-Marcos MA, Gomez-Sanchez L, Patino-Alonso MC, et al. A body shape index and vascular structure and function in Spanish adults (MARK study): $A$ cross-sectional study. Medicine (Baltimore). 2018;97(47):e13299. doi:10. 1097/MD.0000000000013299

8. Shenoy U, Jagadamba. Influence of Central Obesity Assessed by Conicity Index on Lung Age in Young Adults.J Clin Diagn Res. 201 7; 11 (4):CC09-CC12. doi: 10.7860/JCDR/2017/23428.9718
9. Kumar, N., \& Goswami, S. Comparison of Rockport one-mile walk test and McArdle step test for the prediction of $\mathrm{VO}_{2}$ max. Saudi Journal of Sports Medicine 2019;19:82-85.

10. Sacco JJ, Botten J, Macbeth F, Bagust A, Clark P. The Average Body Surface Area of Adult Cancer Patients in the UK: A Multicentre Retrospective Study. PLoS One. 2010; 5 (1): e8933. Bibcode:2010PLoSO...5.8933S. doi:10.1371/ journal. pone.0008933. PMC 2812484.PMID 20126669. Retrieved 2012-09-09.

11. Dybala MP, Brady MJ, Hara M. Disparity in Adiposity among Adults with Normal Body Mass Index and Waist-to-Height Ratio. iScience. 2019;21:612623. doi:10.1016/j.isci.2019.10.062

12. Liu B, Li Y, Guo J, Fan Y, Li L, Li P. Body Mass Index and Its Change from Adolescence to Adulthood Are Closely Related to the Risk of Adult Metabolic Syndrome in China. Int J Endocrinol. 2021;2021:8888862. Published 2021 Feb 18. doi:10.1155/2021/8888862

13. Kuk JL, Saunders TJ, Davidson LE, Ross R. Age-related changes in total and regional fat distribution. Ageing Res Rev. 2009;8(4):339-348. doi:10.1016/ j. arr.2009.06.001

14. Bluhar M, Laufs U. New concepts for body shape related cardiovascular risk: role of fat distribution and adipose tissue functions. Eur Ht J. 2019; 40:2856-8. doi: 10.1093/eurheartj/ehz411

15. Hattiwale HM, Maniyar SA, Das KK, Dhundasi SA. Role of body mass index on physical fitness index in two different Age groups of healthy young males from north interior Karnataka,India. AJMS. 2008; (1): 50-54

16. Radovanovic S, Kocic S, Gajovic G, Radevic S, Milosavljevic M, Niciforovic J The impact of body weight on aerobic capacity. Med Glas (Zenica) 2014;11(1):204-209.

17. Albornoz-Guerrero, J., Zapata-Lamana, R., Reyes-Molina, D., Cigarroa, I. García Pérez de Sevilla, G., \& García-Merino, S. Overweight/Obese Schoolchildren with Low Muscle Strength Have a Lower Cardiorespiratory Capacity and Greater Cardiovascular Risk: Results of the School Health Survey of the Extreme South of Chile 2019. Children (Basel, Switzerland). 2021;8(9):734.https://doi.org/10.3390/children8090734

18. Aubert J D: Peak VO in Obesity: Is It Worth the Effort? Respiration 2017;94:486-487. doi: 10.1159/000481573

19. Salvadori A, Fanari P, Fontana M, et al. Oxygen Uptake and Cardiac Performance in Obese and Normal Subjects during Exercise. Respiration. 1999;66:25-33. doi: 10.1159/000029333 